BMJ Open

Diabetes

Research

\& Care

\title{
Real-world crude incidence of hypoglycemia in adults with diabetes: Results of the InHypo-DM Study, Canada
}

\author{
Alexandria Ratzki-Leewing, ${ }^{1}$ Stewart B Harris, ${ }^{1,2}$ Selam Mequanint, ${ }^{2}$ \\ Sonja M Reichert, ${ }^{2}$ Judith Belle Brown, ${ }^{2}$ Jason Edward Black, ${ }^{1}$ Bridget L Ryan ${ }^{1,2}$
}

To cite: Ratzki-Leewing A, Harris SB, Mequanint S, et al. Real-world crude incidence of hypoglycemia in adults with diabetes: Results of the InHypo-DM Study, Canada. BMJ Open Diab Res Care 2018;6:e000503. doi:10.1136/ bmjdrc-2017-000503

Received 6 December 2017 Revised 23 February 2018 Accepted 10 March 2018
Check for updates

${ }^{1}$ Department of Epidemiology and Biostatistics, Schulich School of Medicine and Dentistry, Western University, London, Ontario, Canada ${ }^{2}$ Department of Family Medicine, Schulich School of Medicine and Dentistry, Western University, London, Ontario, Canada

Correspondence to Dr Stewart B Harris; Stewart.Harris@schulich. uwo.ca

\section{ABSTRACT}

Objective Very few real-world studies have been conducted to assess the incidence of diabetes-related hypoglycemia. Moreover, there is a paucity of studies that have investigated hypoglycemia among people taking secretagogues as a monotherapy or in combination with insulin. Accordingly, our research team developed and validated the InHypo-DM Person with Diabetes Mellitus Questionnaire (InHypo-DMPQ) with the aim of capturing the real-world incidence of self-reported, symptomatic hypoglycemia. The questionnaire was administered online to a national sample of Canadians ( 218 years old) with type 1 diabetes mellitus (T1DM) or type 2 diabetes mellitus (T2DM) treated with insulin and/or insulin secretagogues. Research design and methods Self-report data obtained from the InHypo-DMPQ were descriptively analyzed to ascertain the crude incidence proportions and annualized incidence densities (rates) of 30-day retrospective nonsevere and 1-year retrospective severe hypoglycemia, including daytime and nocturnal events.

Results A total of 552 people (T2DM: 83\%; T1DM: 17\%) completed the questionnaire. Over half $(65.2 \%)$ of the total respondents reported experiencing at least one event (non-severe or severe) at an annualized crude incidence density of 35.1 events per person-year. The incidence proportion and rate of non-severe events were higher among people with T1DM versus T2DM (77\% and 55.7 events per person-year vs $54 \%$ and 28.0 events per person-year). Severe hypoglycemia was reported by $41.8 \%$ of all respondents, at an average rate of 2.5 events per person-year.

Conclusions The results of the InHypo-DMPQ, the largest real-world investigation of hypoglycemia epidemiology in Canada, suggest that the incidence of hypoglycemia among adults with diabetes taking insulin and/or insulin secretagogues is higher than previously thought.

\section{BACKGROUND}

Hypoglycemia is a common adverse event in people with type 1 diabetes mellitus (T1DM) or type 2 diabetes mellitus (T2DM) treated with insulin and/or insulin secretagogues (sulfonylureas or glinides). ${ }^{1-4}$ It is often unpredictable and recurrent, ${ }^{5}$ resulting in potentially severe physical $^{6}$ and psychosocial ${ }^{7}$ morbidity, as well

\section{Significance of this study}

What is already known about this subject?

- Very few real-world studies have been conducted to assess the incidence of diabetes-related hypoglycemia.

- Moreover, there is a paucity of studies that have investigated hypoglycemia among people taking secretagogues as a monotherapy or in combination with insulin.

\section{What are the new findings?}

- A total of 552 people (type 2 diabetes mellitus: 83\%; type 1 diabetes mellitus: $17 \%$ ) completed the questionnaire.

- Over half $(65.2 \%)$ of the total respondents reported experiencing at least one event (non-severe or severe) at an annualized crude incidence density of 35.1 events per person-year.

How might these results change the focus of research or clinical practice?

- The derived estimates underscore the pressing need for improved management strategies and a greater investment in public health initiatives aimed at reducing the human and economic burden of hypoglycemia.

as associated economic burden. ${ }^{8}{ }^{9}$ Hypoglycemia can also be life-threatening, accounting for $6 \%-10 \%$ of all diabetes-related mortalities, according to recent data. ${ }^{10} 11$

To avoid hypoglycemia, many people with T1DM or T2DM will intentionally maintain their plasma glucose levels above recommended values, ultimately increasing their risk of long-term microvascular and macrovascular complications. ${ }^{12}$ This risk is further exacerbated by clinical inertia among healthcare providers (HCPs). ${ }^{13} 14$ A recent study found that nearly $76 \%$ of clinicians would treat their patients with diabetes more aggressively if not for concerns about inducing low blood sugar. ${ }^{15}$ 
Current treatment guidelines and clinical strategies have emphasized the need to balance effective glycemic control with the risk of hypoglycemia. ${ }^{16}$ This focus has been complemented by the increasing availability of safer antihyperglycemic treatments and therapeutic combinations, as well as the integration of technologies such as continuous glucose monitoring ${ }^{17}$ that can minimize the frequency of hypoglycemia events. However, despite the increased recognition of its impact and focus on improving hypoglycemia management strategies, the current burden of hypoglycemia in the real-world context remains unclear. ${ }^{2} 18$

In particular, the putative epidemiology of hypoglycemia has largely depended on the safety results of clinical drug trials, which often exclude individuals who are at greatest risk of hypoglycemia and involve highly controlled settings. Such sampling methods restrict the external validity of trial-based estimates, which, compared with those derived from real-world studies, have been shown to underestimate the true incidence of hypoglycemia. ${ }^{19}$ In comparison, hypoglycemia rates have been examined by observational studies using various methods such as self-report or examination of emergency records, pharmacy records, or claims databases. ${ }^{2}$ While often reporting higher incidence values than trials, ${ }^{20-25}$ some observational studies may still not accurately capture the true extent of population-based hypoglycemia. For example, patient under-reporting ${ }^{2627}$ or poor clinical documentation may lead to an underestimation of the event frequency. ${ }^{22}{ }^{28}$ Conversely, some observational study designs may overestimate the incidence of adverse events; common caveats, according to a study by Mansi (2015), include sampling, response, and surveillance bias, as well as confounding by indication. ${ }^{29}$

Contemporary real-world population-based investigations of hypoglycemia frequencies may help rectify the imprecision related to event estimation, promoting the integration of newer and safer pharmacotherapies in practice, improved clinical interventions, as well as optimal allocation of public health resources. However, within Canada and elsewhere, such evidence is sparse and narrow in scope (ie, restricted by type of diabetes and severity of hypoglycemia). ${ }^{2}$ In particular, very few population-based studies have yet investigated the distribution of hypoglycemia among people taking secretagogues either as a monotherapy or in combination with insulin. ${ }^{30}$

To address these knowledge gaps, the InHypo-DM Person with Diabetes Mellitus Questionnaire (InHypo-DMPQ) was developed and validated to quantify the real-world incidence of self-reported, symptomatic hypoglycemia in Canada: a pragmatic and significant patient-important outcome in the clinical management of diabetes. The questionnaire was administered online to a nationally representative sample of Canadians $(\geq 18$ years old) with T1DM or T2DM treated with insulin and/ or secretagogues.

\section{METHODS}

\section{Study design}

The current evaluation is a subcomponent of the UnderstandINg the impact of HYPOglycemia on Diabetes Management: A Survey of Perspectives and Practices (InHypo-DM Study) - the largest hypoglycemia research program conducted in Canada to date. ${ }^{30-34}$ Specifically, the overarching objectives of the cross-sectional InHypo-DMPQ were threefold: (1) to quantify the complex factors contributing to suboptimal hypoglycemia management behavior (61 items); (2) to ascertain the real-world, self-reported incidence of hypoglycemia (6 items); and (3) to evaluate the sociodemographic and clinical factors associated with hypoglycemia frequency ( 15 items).

This manuscript uses data that were collected for the purposes of objectives 2 and 3 .

\section{Sampling and data collection}

Participants were recruited into the study from a pre-existing online panel of the general public. This panel (i-Say panel) was established and maintained by Ipsos Interactive Services, the largest polling firm in Canada. Multisource recruitment, quota sampling, and quality monitoring were used to ensure that the demographic distribution of the panel sample represented that of the general Canadian population. ${ }^{35}$

The i-Say panel consisted of 133326 members across Canada, from which 2706 individuals were identified on the basis of diabetes status (self-report of T1DM or T2DM) and age ( $\geq 18$ years old); this information was extracted from existing profile records. Randomly selected individuals meeting these entry criteria were subsequently contacted in waves using a convenience sampling approach between 20 November and 2 December 2015. Those interested in participating in the study were asked to complete a brief screening form about their current medication use. Only respondents who self-reported being treated with insulin and/or secretagogues were eligible for inclusion. Literacy in English or French was a requisite for study enrollment; no other exclusion criteria were imposed.

Immediately on confirmation of eligibility, respondents were permitted to access the InHypo-DMPQ via the Ipsos Interactive Services platform. The InHypo-DMPQ was fielded (in English and French) until a prespecified sample size was achieved. Those who completed the questionnaire were offered points by Ipsos Interactive Services; these points could be redeemed for items featured in their rewards catalog.

\section{Measures}

\section{Hypoglycemia frequency}

Respondents self-reported on the number of times they experienced a non-severe or severe hypoglycemia event within the last 30 days or 1 year, respectively, including daytime (occurring during waking hours) and nocturnal (occurring while the respondent was sleeping or attempting to sleep) events. Several 
Table 1 Definitions of hypoglycemia type according to severity and time of event

\begin{tabular}{ll}
\hline $\begin{array}{l}\text { Hypoglycemia } \\
\text { type }\end{array}$ & Definition \\
\hline Non-severe & $\begin{array}{l}\text { A composite of mild and moderate events. } \\
\text { Any self-reported symptomatic event } \\
\text { that could be rectified by self-action. } \\
\text { Symptoms included sweatiness, hunger, } \\
\text { anxiety, weakness, confusion, heart } \\
\text { palpitations, difficulty speaking, and/or loss } \\
\text { of concentration that could be treated by } \\
\text { the individual. }\end{array}$ \\
Severe & $\begin{array}{l}\text { Any self-reported event that absolutely } \\
\text { required third-party assistance in the } \\
\text { administration of treatment (eg, glucose or } \\
\text { glucagon). }\end{array}$ \\
Daytime & $\begin{array}{l}\text { Any event that occurred during normal } \\
\text { waking hours. }\end{array}$ \\
Nocturnal & $\begin{array}{l}\text { Any event that occurred while the } \\
\text { respondent was sleeping or attempting to } \\
\text { sleep. }\end{array}$ \\
\hline
\end{tabular}

strategies were also implemented to promote open and honest responses among participants; specifically, the questionnaire was self-administered, completed outside of the clinical context (as opposed to during a scheduled medical appointment), and all answers were anonymized.

Standardized, clear, and accessible definitions of hypoglycemia (table 1), consistent with the Canadian Diabetes Association Clinical Practice Guidelines, ${ }^{36}$ were provided to ensure a thorough understanding among respondents and to establish uniformity throughout the study. The recall periods for daytime and nocturnal non-severe and severe hypoglycemia were determined by experts and in compliance with the patient-report outcomes guidance sanctioned by the Food and Drug Administration. ${ }^{37}$ Specification of each interval length accounted for the episodic and highly acute nature of hypoglycemia, variations in severity, as well as the need to balance the risk of recall bias with the sensitivity of the outcome measure. The selected recall periods were also commonly cited for self-reported events in the literature. ${ }^{19}$

\section{Sociodemographic and clinical characteristics}

Respondents self-reported various sociodemographic and clinical characteristics. Five diabetes-specific questions captured the respondent's diabetes type, duration of diabetes, most recent hemoglobin Alc (HbAlc), number of insulin injections per day, and presence of comorbidities. Additionally, nine sociodemographic questions pertained to the respondent's gender, age, location of residence, drug coverage, living arrangements, employment status, income, and education level. All characteristics were assessed at the time of questionnaire completion.

\section{Sample size}

The sample size of 552 respondents was based on objective 1 of the InHypo-DMPQ (refer to the Study design section). ${ }^{38}$

\section{Analysis}

Questionnaire data were descriptively analyzed using STATA V.13.0. Data were tested for feasibility and completeness prior to analysis. Categorical data were summarized as proportions with CIs, while continuous data as mean and SD or median and IQR.

Daytime and nocturnal crude estimates of incidence proportion and annualized incidence densityrate were calculated for (1) total (a composite of all non-severe and severe events), (2) non-severe, and (3) severe hypoglycemia. Incidence estimates were also calculated according to diabetes type.

For each endpoint, the crude incidence proportion was calculated as the percentage of participants who self-reported experiencing at least one hypoglycemia event during the specified retrospective time period. Thus, all reported incidence proportions pertained to the recall period in question.

Crude incidence densities were calculated as the total number of hypoglycemia events divided by the total number of years under study contributed by all study participants. All crude incidence densities were annualized (ie, reported as events per person-year) to facilitate comparison across different severities of hypoglycemia. The associated $95 \%$ CIs for both types of incidence estimations were computed.

\section{RESULTS}

\section{Respondents' characteristics}

A total of 552 people (T2DM: 83\%; T1DM: 17\%) completed the questionnaire between 20 November and 2 December 2015. Table 2 provides a summary of all respondent characteristics. Data were missing from non-severe daytime at $0.2 \%$, severe daytime at $0.2 \%$, and severe nocturnal at $0.4 \%$. Complete-case analysis was used to account for missing data. The mean age of the respondents was 51.5 (SD: 15.3) years old and 56\% were male. The mean duration of diabetes was 13.2 (SD: 9.8 ) years. Among study participants, $46 \%$ reported that they were taking insulin, 39\% secretagogues, and $15 \%$ a combination of the two therapies.

People with T1DM were younger than people with T2DM (42.5 and 53.4 years old $(\mathrm{P}<0.001)$, respectively) and reported nearly two times the duration of diabetes (T1DM: 20.3 years; T2DM: 11.7 years; $\mathrm{P}<0.001$ ). The median HbAlc across the sample was $7.2 \%$ (IQR: $6 \%-9 \%)$ - only a slightly higher HbA1c was reported for T1DM (T1DM: $7.7 \%$ (IQR: $6.5 \%-9.7 \%$ ) vs T2DM: $7.1 \%$ (IQR: $6.3 \%-8.7 \%)$ ), a difference that was not statistically significant $(\mathrm{P}=0.47)$. Total, severe, and non-severe hypoglycemia are presented below for the overall study sample and according to type of diabetes. 
Table 2 Characteristics of the InHypo-DMPQ respondents

\begin{tabular}{|c|c|c|c|c|}
\hline Characteristics & Categories & Overall $(n=552)$ & $\begin{array}{l}\text { T1DM } \\
(\mathrm{n}=94)\end{array}$ & $\begin{array}{l}\text { T2DM } \\
(n=458)\end{array}$ \\
\hline Age, mean (SD) & & $51.5(15.3)$ & $42.5(14.5)$ & $53.4(14.8)$ \\
\hline Diabetes duration, median (IQR) & & $10.0(5.0-19.0)$ & $16(8.0-32.0)$ & $10.0(5.0-16.0)$ \\
\hline Gender, n (\%) & Female & $243(44.0)$ & $44(46.8)$ & $199(43.4)$ \\
\hline HbA1c, median (IQR) & & $7.2(4.0-9.0)$ & $7.7(6.5-9.6)$ & $7.1(6.3-8.7)$ \\
\hline \multirow[t]{2}{*}{ Drug coverage, n (\%) } & Self-pay & $51(9.2)$ & $12(12.8)$ & $40(8.7)$ \\
\hline & Other (eg, public, private) & $500(90.7)$ & $82(87.2)$ & $418(91.3)$ \\
\hline \multirow[t]{2}{*}{ Full-time work status, n (\%) } & Yes & $235(42.6)$ & $54(57.4)$ & $181(39.5)$ \\
\hline & $\begin{array}{l}\text { Other (eg, part-time/seasonal, } \\
\text { student, stay-at-home parent, } \\
\text { disability, retired, unemployed) }\end{array}$ & $317(57.4)$ & $40(42.6)$ & $277(60.5)$ \\
\hline Shift-work status, n (\%) & Yes & $121(21.9)$ & $31(33.0)$ & $90(19.7)$ \\
\hline CGM use, $\mathrm{n}(\%)$ & Yes & $201(36.4)$ & $32(34.0)$ & $169(37.0)$ \\
\hline Comorbidity, n (\%) & Yes & $129(23.4)$ & $18(19.1)$ & $111(24.2)$ \\
\hline Medication type, n (\%) & $\begin{array}{l}\text { Insulin secretagogues alone } \\
\text { Insulin alone } \\
\text { Insulin+insulin secretagogues }\end{array}$ & $\begin{array}{l}217(39.3) \\
253(45.8) \\
82(14.9)\end{array}$ & $\begin{array}{l}0(0) \\
94(100) \\
0(0)\end{array}$ & $\begin{array}{l}217(47.4) \\
159(34.7) \\
82(17.9)\end{array}$ \\
\hline Region, n (\%) & $\begin{array}{l}\text { Urban } \\
\text { Rural }\end{array}$ & $\begin{array}{l}485(87.9) \\
67(12.1)\end{array}$ & $\begin{array}{l}87(92.6) \\
7(7.4)\end{array}$ & $\begin{array}{l}398(86.9) \\
60(13.1)\end{array}$ \\
\hline \multirow[t]{2}{*}{ Main HCP type, n (\%) } & Family physician & $367(66.5)$ & $49(52.1)$ & $318(69.4)$ \\
\hline & $\begin{array}{l}\text { Other (eg, nurse practitioner, } \\
\text { endocrinologist, pharmacist, } \\
\text { diabetes specialist, diabetes nurse, } \\
\text { internist) }\end{array}$ & $185(33.5)$ & $45(47.9)$ & $140(30.6)$ \\
\hline \multirow[t]{2}{*}{ Education status, n (\%) } & $\begin{array}{l}\text { Elementary school, high school, } \\
\text { CEGEP }\end{array}$ & $184(33.3)$ & $27(28.7)$ & $157(34.3)$ \\
\hline & $\begin{array}{l}\text { Other (eg, technical school/college, } \\
\text { university, graduate/professional } \\
\text { school) }\end{array}$ & $368(66.7)$ & $67(71.3)$ & $301(65.7)$ \\
\hline \multirow[t]{2}{*}{ Living arrangements, n (\%) } & Alone & $171(31.0)$ & $23(24.5)$ & $148(32.3)$ \\
\hline & $\begin{array}{l}\text { Other (eg, spouse, family member, } \\
\text { roommate/boarder) }\end{array}$ & $381(69.0)$ & $71(75.5)$ & $310(67.7)$ \\
\hline \multirow[t]{4}{*}{ Income, n (\%) } & Less than $\$ 30000$ & $141(25.5)$ & $19(20.2)$ & $128(28.1)$ \\
\hline & $\$ 30000-\$ 50000$ & $120(21.7)$ & $26(27.7)$ & $94(20.6)$ \\
\hline & $\$ 51000-\$ 75000$ & $115(20.8)$ & $21(22.3)$ & $94(20.6)$ \\
\hline & More than $\$ 75000$ & $167(30.3)$ & $28(29.8)$ & $139(30.5)$ \\
\hline
\end{tabular}

CGM, continuous glucose monitoring; HbA1c, hemoglobin A1c; HCP, healthcare provider; InHypo-DMPQ, InHypo-DM Person with Diabetes Mellitus Questionnaire; T1DM, type 1 diabetes mellitus; T2DM, type 2 diabetes mellitus.

Participants primarily resided in Ontario (41.5\%) or Quebec (21.7\%): proportions that reflect the realworld province-based distributions $(42.9 \%$ and $23.3 \%$, respectively). ${ }^{39}$ As well, the percentages of individuals from Alberta (10.5\%), British Columbia (9.4\%), and Manitoba/Saskatchewan (7.6\%) were also fairly comparable with province-level population data, with reported diabetes frequencies of $8.6 \%, 10.8 \%$, and $8.5 \%$, respectively. ${ }^{39}$ The proportion of respondents from the Atlantic provinces (Newfoundland and Labrador, Prince Edward Island, Nova Scotia, and New Brunswick) was 5\% lower $(14.1 \%$ vs $9.1 \%)$ than the actual provincial estimate. ${ }^{39}$ Not surprisingly, very little data were collected from the territories.

\section{Frequency estimates}

\section{Total hypoglycemia}

Over half $(65.2 \%$ (95\% CI $61.0 \%$ to $69.0 \%)$ ) of the total sample reported experiencing at least one hypoglycemia event of any type within 30 days for non-severe and 1 year for severe hypoglycemia. On average, respondents reported an annualized crude incidence density of 35.1 (95\% CI 34.6 to 35.6) events per person-year. Respondents with at least one event $(n=360)$ typically reported experiencing both daytime and nocturnal hypoglycemia (58.3\% (95\% CI $53.2 \%$ to $63.3 \%)$ ).

Among respondents with T1DM, 83.0\% (95\% CI 74.1\% to $89.2 \%$ ) had experienced at least one hypoglycemia event. These individuals reported an overall annualized 
Table 3 Crude incidence proportions and incidence densities by type of diabetes and hypoglycemia event

\begin{tabular}{|c|c|c|c|}
\hline & All respondents & T1DM & T2DM \\
\hline & $n=552$ & $\mathrm{n}=94$ & $n=456$ \\
\hline \multicolumn{4}{|l|}{ Total hypoglycemia } \\
\hline \multicolumn{4}{|l|}{ Overall } \\
\hline Incidence proportion, \% (95\% Cl) & 65.2 (61.0 to 69.0$)$ & 83.0 (74.1 to 89.2$)$ & $62.0(56.9$ to 65.8$)$ \\
\hline Incidence density, events PPY $(95 \% \mathrm{Cl})$ & 35.1 (34.6 to 35.6$)$ & 58.1 (56.6 to 59.7$)$ & $30.4(29.9$ to 30.9$)$ \\
\hline \multicolumn{4}{|l|}{ Daytime } \\
\hline Incidence proportion, \% (95\% Cl) & 65.5 (58.2 to 66.3$)$ & $80.0(70.6$ to 86.7$)$ & 59.0 (54.2 to 63.2$)$ \\
\hline Incidence density, events PPY $(95 \% \mathrm{Cl})$ & 22.7 (22.2 to 23.0$)$ & 43.4 (42.0 to 44.7$)$ & $18.3(17.9$ to 18.7$)$ \\
\hline \multicolumn{4}{|l|}{ Nocturnal } \\
\hline Incidence proportion, \% (95\% Cl) & 41.1 (36.9 to 45.1$)$ & $63.0(52.7$ to 71.9$)$ & 36.7 (32.1 to 40.9$)$ \\
\hline Incidence density, events PPY $(95 \% \mathrm{Cl})$ & 12.6 (12.3 to 12.9$)$ & $14.7(14.0$ to 15.5$)$ & $12.1(11.8$ to 12.4$)$ \\
\hline \multicolumn{4}{|l|}{ Non-severe hypoglycemia } \\
\hline \multicolumn{4}{|l|}{ Overall } \\
\hline Incidence proportion, \% (95\% Cl) & 58.2 (53.8 to 62.0$)$ & $77.0(67.1$ to 84.0$)$ & $54.0(49.6$ to 58.7$)$ \\
\hline Incidence density, events PPY $(95 \% \mathrm{Cl})$ & 32.7 (32.2 to 33.2$)$ & 55.7 (54.2 to 57.2$)$ & $28.0(27.5$ to 28.4$)$ \\
\hline \multicolumn{4}{|l|}{ Daytime } \\
\hline Incidence proportion, \% (95\% Cl) & 55.4 (51.3 to 59.6$)$ & 74.5 (64.8 to 82.2$)$ & $52.0(47.0$ to 56.1$)$ \\
\hline Incidence density, events PPY $(95 \% \mathrm{Cl})$ & 21.0 (20.7 to 21.5$)$ & 41.8 (40.5 to 43.1$)$ & $16.8(16.4$ to 17.2$)$ \\
\hline \multicolumn{4}{|l|}{ Nocturnal } \\
\hline Incidence proportion, \% (95\% Cl) & 33.9 (30.0 to 37.9$)$ & 54 (44.2 to 64.0$)$ & 29.7 (25.6 to 34.0$)$ \\
\hline Incidence density, events PPY (95\% Cl) & $11.6(11.3$ to 11.9$)$ & $13.9(13.2$ to 14.7$)$ & 11.1 (10.8 to 11.4$)$ \\
\hline \multicolumn{4}{|l|}{ Severe hypoglycemia } \\
\hline \multicolumn{4}{|l|}{ Overall } \\
\hline Incidence proportion, \% (95\% Cl) & 40.4 (36.5 to 44.7$)$ & 54.3 (44.2 to 64.0$)$ & 38.0 (33.4 to 42.3$)$ \\
\hline Incidence density, events PPY $(95 \% \mathrm{Cl})$ & 2.5 (2.3 to 2.6$)$ & 2.4 (2.1 to 2.8$)$ & 2.5 (2.3 to 2.6$)$ \\
\hline \multicolumn{4}{|l|}{ Daytime } \\
\hline Incidence proportion, \% (95\% Cl) & 38.2 (34.0 to 42.1$)$ & 52.1 (42.2 to 61.9$)$ & 35.1 (30.9 to 39.6) \\
\hline Incidence density, events PPY $(95 \% \mathrm{Cl})$ & 1.5 (1.4 to 1.6$)$ & $1.6(1.4$ to 1.9$)$ & 1.5 (1.4 to 1.6$)$ \\
\hline \multicolumn{4}{|l|}{ Nocturnal } \\
\hline Incidence proportion, \% (95\% Cl) & $27.2(23.4$ to 30.8$)$ & 34.0 (25.3 to 44.1$)$ & 25.4 (21.7 to 29.6$)$ \\
\hline Incidence density, events PPY $(95 \% \mathrm{Cl})$ & $1.0(0.9$ to 1.0$)$ & $0.8(0.6$ to 1.0$)$ & $1.0(0.9$ to 1.1$)$ \\
\hline
\end{tabular}

Incidence proportion refers to 30-day incidence for non-severe hypoglycemia and 1-year incidence for severe hypoglycemia.

PPY, per person-year; T1DM, type 1 diabetes mellitus; T2DM, type 2 diabetes mellitus.

hypoglycemia rate of 58.1 (95\% CI 56.6 to 59.7 ) events per person-year. Among those with T1DM who experienced hypoglycemia $(\mathrm{n}=78)$, most $(72 \%(95 \%$ CI $61.1 \%$ to $80.6 \%$ ) ) reported both a daytime and nocturnal event.

Hypoglycemia was also common among people with T2DM; approximately $62.0 \%$ (95\% CI $56.9 \%$ to $65.8 \%$ ) of individuals experienced at least one hypoglycemia event at a rate of 30.4 (95\% CI 29.9 to 30.9) events per person-year. Over half ( $55.0 \%$ (95\% CI $49.1 \%$ to $60.7 \%)$ ) of respondents who reported at least one event $(\mathrm{n}=280)$ reported experiencing both daytime and nocturnal hypoglycemia.

\section{Non-severe hypoglycemia}

Non-severe hypoglycemia affected 58.2\% (95\% CI 53.8\% to $62.0 \%$ ) of all respondents in the 30 days preceding questionnaire completion. Across all individuals, 18012 events were reported, resulting in an annualized incidence density of 32.7 (95\% CI 32.2 to 33.2) events per person-year. Furthermore, the incidence density of nocturnal hypoglycemia was approximately 20 times higher among individuals who reported having at least 1 daytime hypoglycemia event when compared with those who reported zero daytime hypoglycemia events (0.9 (95\% CI 0.8 to 1.1 ) vs 20.2 (95\% CI 19.7 to 20.7 ) events per person-year, respectively).

Seventy-seven percent (95\% CI $67.1 \%$ to $84.0 \%$ ) of respondents with T1DM reported experiencing a non-severe hypoglycemia event (daytime or nocturnal) within the past 30 days. Respondents reported on average 55.7 (95\% CI 54.2 to 57.2) non-severe hypoglycemia events per person-year. The percentage of individuals who reported 
both a daytime and nocturnal non-severe event was 25 times higher than the percentage of respondents who reported a nocturnal event alone $(52.1 \%(95 \%$ CI $42.2 \%$ to $61.9 \%$ ) vs $2.1 \%$ (95\% CI $0.6 \%$ to $7.4 \%)$ ). The incidence density of non-severe nocturnal hypoglycemia was 18.3 (95\% CI 17.4 to 19.4) events per person-year among those who reported at least one non-severe daytime event, as compared with 1 (95\% CI 0.6 to 1.5 ) event per personyear among those who reported no daytime events.

A smaller proportion $(54.0 \%$ (95\% CI $49.6 \%$ to $58.7 \%)$ ) of individuals with T2DM, as compared with T1DM, experienced a non-severe event (daytime or nocturnal) in the past 30 days. Despite the fact that the absolute number of non-severe events was substantially higher in people with T2DM (T2DM: 12 780; T1DM: 5232), the incidence density among people with T2DM was near half that observed in T1DM at 28.0 (95\% CI 27.5 to 28.4) events per person-year. Within the people with T2DM, nocturnal hypoglycemia occurred far more frequently among those who also reported at least one non-severe daytime event (20.7 (95\% CI 20.2 to 21.3 ) vs 0.9 (95\% CI 0.8 to 1.1 ) events per person-year, respectively).

\section{Severe hypoglycemia}

Two hundred and twenty-three $(40.4 \%$ (95\% CI $36.5 \%$ to $44.7 \%)$ ) respondents reported experiencing at least one severe hypoglycemia event in the year preceding the completion of the questionnaire. On average, 2.5 (95\% CI 2.3 to 2.6) severe events per person-year were reported. Among those who experienced a severe event, most (60\% (95\% CI $53.5 \%$ to $66.3 \%)$ ) reported experiencing both a daytime and nocturnal event in the past year. Finally, the 1-year incidence proportion and incidence density of severe hypoglycemia was substantially higher among those who reported at least one non-severe event (daytime or nocturnal) versus zero non-severe events $(57.9 \%$ (95\% CI $52.2 \%$ to $63.0 \%)$ and $3.9(95 \%$ CI 3.7 to 4.2 ) events per person-year vs $16.9 \%$ (95\% CI $12.6 \%$ to $22.3 \%)$ and 0.4 (95\% CI 0.3 to 0.5 ) events per person-year, respectively).

Over half $(54.3 \%$ (95\% CI $44.2 \%$ to $64.0 \%))$ of respondents with T1DM experienced at least one severe hypoglycemia event in the previous year. Among these individuals, a total of 228 severe events were reported, translating to an incidence density of 2.4 (95\% CI 2.1 to 2.8 ) events per person-year. Among those with at least one reported non-severe event (daytime or nocturnal) in the past 30 days, the majority $(62.5 \%$ (95\% CI $51.0 \%$ to $72.8 \%)$ ) also experienced a severe event in the past year; additionally, the rate of severe hypoglycemia was approximately 8.5 times higher when compared with those with zero reported non-severe events (3.1 (95\% CI 2.7 to 3.5 ) vs 0.4 (95\% CI 0.2 to 0.7 ) events per person-year, respectively).

While the 1-year incidence proportion of severe hypoglycemia was lower among individuals with T2DM $(38.0 \%$ (95\% CI $33.4 \%$ to $42.3 \%)$ ) than with T1DM, the incidence density was slightly higher $(2.5$ (95\% CI 2.3 to 2.6) events per person-year) in individuals with T2DM.
Estimates of incidence density of severe hypoglycemia in those who reported at least one hypoglycemia event (severe or non-severe) were higher in those with T2DM (4.0 (95\% CI 3.8 to 4.2 ) events per person-year) than T1DM (2.9 (95\% CI 2.6 to 3.3) events per person-year). As well, the rate of severe hypoglycemia in people with T2DM was approximately 11 times higher in individuals who reported at least 1 daytime or nocturnal non-severe event (4.2 (95\% CI 4.0 to 4.5$)$ vs 0.4 (95\% CI 0.3 to 0.5$)$ events per person-year, respectively) (Table 3 ).

\section{DISCUSSION}

This real-world study aimed to assess the crude incidence proportions and annualized incidence densities of daytime and nocturnal non-severe and severe hypoglycemia in a representative sample of Canadians $(\geq 18$ years old) at risk for hypoglycemia. The population-based frequency of hypoglycemia was high across all respondents, with close to two-thirds of the respondents reporting at least one non-severe (in the last 30 days) or severe (in the last year) hypoglycemia event. Daytime events were typically more common than nocturnal events, both in terms of the percentage of individuals affected (incidence proportion) and rate of occurrence (incidence density); those who reported a daytime event were more likely to have also reported a nocturnal event.

As expected, event frequencies were generally higher among respondents with T1DM as compared with those with T2DM. Nevertheless, the burden of hypoglycemia was substantial in people with T2DM with well over half of respondents reporting at least one event, at an annualized rate of 14.7 events per person-year. Indeed, while incidence densities were typically higher among people with T1DM when compared with people with T2DM, the absolute number of events was considerably higher for people with T2DM. A recent study that summarized the economic burden of hypoglycemia across 14 studies in the USA found that severe hypoglycemia requiring non-medical assistance resulted in higher indirect costs among patients with T2DM than in patients with T1DM (\$579 vs \$242). ${ }^{40}$ The substantial absolute burden of hypoglycemia in T2DM, which is well supported in the literature ${ }^{49}$ is thus especially important for future public health strategies.

Rates of severe hypoglycemia were also markedly high, particularly among respondents who had also reported at least one non-severe event. Interestingly, people with T2DM reported slightly higher rates of severe hypoglycemia when compared with those with T1DM, despite the fact that relatively fewer individuals with T2DM actually experienced an event. In fact, among those reporting any event, the incidence rate of severe hypoglycemia was approximately $37 \%$ higher in people with T2DM than with T1DM. This observation may allude to the substantial challenges faced by people with T2DM when managing their hypoglycemia and, compared with their T1DM counterpart, signal important deficiencies in management behaviors and/or clinical support. 
The present results generally exceed the frequency estimates derived from traditional glucose-lowering clinical trials. This trend aligns with a recent review, which found hypoglycemia to be more common in real-world contexts than trial-based settings. ${ }^{19}$ Not surprisingly, in contrast with other Canadian-based observational investigations of hypoglycemia epidemiology, the results of this study are relatively more comparable, with main differences likely attributable to variations in hypoglycemia measurement (ie, scope, definitions, and data collection) or sampling frame/methodology. For example, the Canadian cohort of the Hypoglycemia Assessment Tool program ${ }^{41}$ assessed severe hypoglycemia 6 months retrospectively and 4 weeks prospectively. These data collection periods may not have been sufficiently sensitive to capture severe hypoglycemia (a rare event), potentially resulting in an underestimation of annualized incidence densities. Additionally, the Canadian cohort of the Global Attitude Patients and Physicians 2 study $^{20}$ included only 156 individuals with T2DM (excluding people with T1DM) over the age of 40; the use of a highly explanatory sampling frame (ie, patients attending diabetes specialist clinics) was also noted in an investigation by Leiter et al. ${ }^{15}$ Furthermore, these studies commonly relied on patient self-report that was obtained during a scheduled medical appointment with an HCP. ${ }^{15}$

Importantly, none of these Canadian-based studies specifically investigated the distribution of hypoglycemia among people taking secretagogues (alone or in combination with insulin), despite the known potential of these medications to induce hypoglycemia; in fact, people taking sulfonylureas have been shown to experience more severe and longer events when compared with insulin-treated patients. ${ }^{42}$ Globally, the relevance of secretagogues to real-world hypoglycemia frequency has also been only minimally investigated. ${ }^{2}$ The present analysis thus makes an important contribution to both the national and international body of literature around hypoglycemia, offering key insight into the potential choice and individualization of therapeutic options most commonly used in the clinical management of T2DM.

As expected, the present results are on average higher than those reported in most clinical trials. A systematic review, which examined the incidence of hypoglycemia among patients receiving sulfonylureas within randomized controlled trials, found that $10.1 \%$ (95\% CI $7.3 \%$ to $13.8 \%$ ) had at least one mild or moderate hypoglycemic event and $0.8 \%$ (95\% CI $0.5 \%$ to $1.3 \%)$ experienced at least one severe hypoglycemia event. ${ }^{44}$

Alternatively, a more appropriate comparison with other population-based studies suggests that our incidence estimates are reasonably consistent with what is currently published. A recent systematic review and meta-analysis of 46 population-based investigations of hypoglycemia frequency among people with T2DM receiving insulin and oral therapies presented pooled estimates of hypoglycemia incidence proportion and rate similar to those presented in the current analysis ${ }^{2}$ : non-severe (mild/moderate) incidence proportion was slightly lower ( $45 \%$ (95\% CI $34 \%$ to $57 \%)$ compared with $52 \%(95 \%$ CI $47.0 \%$ to $56.1 \%$ ) (daytime non-severe)); non-severe (mild/moderate) incidence rate was similar (19 (95\% CI 0.00 to 51.08) events per person-year compared with 16.82 (95\% CI 16.44 to 17.20 ) events per person-year (daytime non-severe)); and severe incidence rate was approximately similar $(0.80$ (95\% CI 0.00 to 2.15) events per person-year compared with 1.48 (95\% CI 1.37 to 1.59$)$ ). However, severe hypoglycemia incidence proportion was much lower $(6 \%$ (95\% CI $5 \%$ to $7 \%$ ) compared with $35.1 \%$ (30.9\% to $39.6 \%$ ) (daytime severe)); this discrepancy may be explained by the large number of studies that relied on patient reporting and clinical documentation, which have been shown to underestimate hypoglycemia incidence, ${ }^{19}$ as well as the large number of investigations stemming from patient registries. The InHypo-DM study presents a comprehensive, population-based examination of hypoglycemia, including daytime, nocturnal, non-severe, and severe hypoglycemia, that allows for comparison of both incidence proportion and incidence rate.

There are possible limitations associated with our study. First, selection bias may have arisen to the extent that study respondents (ie, individuals who had access to an internet-linked device, who belonged to the i-Say panel with self-reported diagnosis of diabetes, and who were sufficiently interested/motivated to complete the InHypo-DMPQ) differed from the general Canadian population with diabetes at risk for hypoglycemia in ways that could have influenced the self-reported number of hypoglycemia events. Nevertheless, the rigorous sampling and recruitment strategies employed by Ipsos Interactive Services ${ }^{35}$ as well as the high internet uptake in Canada (ranging between $77 \%$ and $87 \%)^{45}$ may have helped mitigate the degree of non-response and coverage bias. It must also be noted, by virtue of the chosen sampling strategy, that the present study demonstrated impressive feasibility. All data were collected, cleaned, and sent to our research center within 10 days of questionnaire administration.

Second, primarily due to issues of practicality, data on non-severe asymptomatic events were not collected. This could have led to an underestimation of respondents' true hypoglycemia frequency. Notwithstanding, the outcome measures, based on participant self-report, employed in the current study encompassed several pragmatic classifications of hypoglycemia considered important in the clinical management of diabetes: documented symptomatic hypoglycemia, probable symptomatic hypoglycemia, as well as pseudo-hypoglycemia. ${ }^{46}$ Certainly, symptomatic hypoglycemia, in the absence of plasma glucose values, can serve as a reasonable proxy for a clinical diagnosis of an event, given that the risks associated with untreated hypoglycemia outweigh those of unnecessary treatment. ${ }^{18}$ Moreover, it is not unreasonable to presume that some participants in our study did in fact corroborate their symptomatic hypoglycemia with a confirmed blood glucose test, lending further reliability 
to the derived estimates; a recent global study of patients with $\mathrm{T} 1 \mathrm{DM}$ or $\mathrm{T}_{2} \mathrm{DM}^{47}$ found that $44.6 \%$ of all participants defined hypoglycemia by using both symptoms and blood glucose measurements rather than by symptoms alone.

Third, self-reported event data may have been subject to recall bias, especially among older individuals who are more likely to suffer from impaired hypoglycemia awareness. ${ }^{48}$ To reduce this potential risk, measures were taken to ensure that all selected recall intervals optimized respondents' abilities to remember events accurately. Participants could also take as much time as necessary to reflect on each item or review clinical documentation (eg, personal glucose records) before providing an answer. Furthermore, it is important to note that estimates of self-reported severe hypoglycemia tend to be rather reliable, given the saliency and relative infrequency of events. ${ }^{49} 50$ One study, which investigated a cohort of insulin-treated patients with T1DM, found that nearly $90 \%$ of participants correctly recalled whether they had a severe hypoglycemia event in the previous year. ${ }^{51}$

Fourth, some characteristics that may influence risk of hypoglycemia were not explicitly captured in the current questionnaire and therefore could not be summarized descriptively (ie, oral agent class or list of comorbidities). However, apart from including patients over 18 on insulin and/or secretagogues, no exclusion criteria were used; therefore, this study aimed to provide an accurate and pragmatic representation of Canadians with diabetes at risk of hypoglycemia and their crude event frequencies. Lastly, adjusted frequency estimates may be informative when attempting to clarify differences in risk among people with diabetes; however, such analyses are beyond the scope of this manuscript. Rather this study draws attention to the alarming degree to which hypoglycemia affects at-risk people with diabetes, an understanding that is critical to effective public health planning and clinical management.

\section{CONCLUSIONS}

The results of the InHypo-DMPQ, the largest real-world investigation of hypoglycemia epidemiology in Canada, suggest that the incidence of hypoglycemia among adults with diabetes taking insulin and/or secretagogues is higher than previously thought-especially among respondents with T2DM. On average, while rates of hypoglycemia were greater in people with T1DM as compared with T2DM, the absolute number of events was substantially lower. Notably, the incidence rate of overall severe hypoglycemia was highest in people with T2DM. The derived estimates underscore the pressing need for improved management strategies and a greater investment in public health initiatives aimed at reducing the human and economic burden of hypoglycemia.
Contributors AR-L and SBH contributed equally to the development of this manuscript as lead authors; specifically, they conceived and designed the work and were involved in data collection, data analysis and interpretation, drafting of the article, critical revision of the article, and final approval of the version to be published. SM, SMR, JBB, and BLR were involved in the conception and design of the work, data collection, data analysis and interpretation, critical revision of the article, and final approval of the version to be published. JEB was involved in the data analysis and interpretation, critical revision of the article, and final approval of the version to be published. All authors have agreed to be accountable for all aspects of the work.

Funding Funding for this study was provided through an investigator-initiated grant from Sanofi Canada.

Competing interests SBH: Sanofi: grant, personal fees, member advisory board, consultant; Eli Lilly: grant, personal fees, member advisory board, consultant, clinical studies; Novo Nordisk: grant, personal fees, member advisory board, consultant, clinical studies; Janssen: grant, personal fees, member advisory board, consultant; Merck: personal Fees, member advisory board, consultant; AstraZeneca: grant, personal fees, member advisory board, consultant, clinical studies; Abbott: grant, personal fees, member advisory board, consultant; Boehringer Ingelheim: grant, personal fees, member advisory board, consultant, clinical studies; JDRF: grant; Lawson: grant; Medtronic: personal fees, member advisory board; Amgen: personal fees, member advisory board; Health Canada/ First Nations and Inuit Health Branch: grant. SMR: Sanofi: grant, coinvestigator for clinical trials (but with no direct monies for me), fees for presentations; AstraZeneca: grant, coinvestigator for clinical trials (but with no direct monies for me), fees for presentations; Novo Nordisk: grant, coinvestigator of clinical trials, with travel for meetings (but no direct grant monies for me), fees paid for presentations; Boehringer Ingelheim: grant, coinvestigator for clinical trials (but with no direct monies for me), fees for presentations; Servier: grant, coinvestigator of clinical trials, with travel for meetings (but no direct grant monies for me), fees paid for presentations; Janssen: fees paid for presentations; Eli Lilly: fees paid for presentations; Merck: fees paid for presentations; Abbott: fees paid for presentations.

Patient consent Obtained.

Ethics approval Ethics approval for the conduct of this study was obtained from the Western University Health Sciences Research Ethics Boards (REB\# 105992 - 16 December 2014).

Provenance and peer review Not commissioned; externally peer reviewed. Data sharing statement No additional data are available.

Open Access This is an Open Access article distributed in accordance with the Creative Commons Attribution Non Commercial (CC BY-NC 4.0) license, which permits others to distribute, remix, adapt, build upon this work non-commercially, and license their derivative works on different terms, provided the original work is properly cited and the use is non-commercial. See: http://creativecommons.org/ licenses/by-nc/4.0/

C Article author(s) (or their employer(s) unless otherwise stated in the text of the article) 2018. All rights reserved. No commercial use is permitted unless otherwise expressly granted.

\section{REFERENCES}

1. Cryer P. Hypoglycemia: pathophysiology, diagnosis, and treatment. New York: Oxford University Press, 1997

2. Edridge CL, Dunkley AJ, Bodicoat DH, et al. Prevalence and Incidence of Hypoglycaemia in 532,542 People with Type 2 Diabetes on Oral Therapies and Insulin: a systematic review and metaanalysis of population based studies. PLoS One 2015;10:e0126427.

3. Bodmer M, Meier C, Krähenbühl S, et al. Metformin, sulfonylureas, or other antidiabetes drugs and the risk of lactic acidosis or hypoglycemia: a nested case-control analysis. Diabetes Care 2008;31:2086-91.

4. UK Hypoglycaemia Study Group. Risk of hypoglycaemia in types 1 and 2 diabetes: effects of treatment modalities and their duration. Diabetologia 2007;50:1140-7.

5. Cryer PE. The barrier of hypoglycemia in diabetes. Diabetes 2008;57:3169-76.

6. Cryer P. Hypoglycaemia: The limiting factor in the glycaemic management of Type I and Type II Diabetes*. Diabetologia 2002;45:937-48.

7. Peyrot M, Rubin RR, Lauritzen T, et al. Psychosocial problems and barriers to improved diabetes management: results of the Cross- 
National Diabetes Attitudes, Wishes and Needs (DAWN) Study. Diabet Med 2005;22:1379-85.

8. Lundkvist $\mathrm{J}$, Berne $\mathrm{C}$, Bolinder $\mathrm{B}$, et al. The economic and quality of life impact of hypoglycemia. Eur J Health Econ 2005;6:197-202.

9. Holstein A, Plaschke A, Egberts EH. Incidence and costs of severe hypoglycemia. Diabetes Care 2002;25:2109-10.

10. Feltbower RG, Bodansky HJ, Patterson CC, et al. Acute complications and drug misuse are important causes of death for children and young adults with type 1 diabetes: results from the Yorkshire Register of diabetes in children and young adults. Diabetes Care 2008;31:922-6.

11. Skrivarhaug T, Bangstad HJ, Stene LC, et al. Long-term mortality in a nationwide cohort of childhood-onset type 1 diabetic patients in Norway. Diabetologia 2006;49:298-305.

12. Amiel SA, Dixon T, Mann R, et al. Hypoglycaemia in Type 2 diabetes. Diabet Med 2008;25:245-54.

13. Dailey GE. Early insulin: an important therapeutic strategy. Diabetes Care 2005;28:220-1.

14. Asche C, LaFleur J, Conner C. A review of diabetes treatment adherence and the association with clinical and economic outcomes. Clin Ther 2011;33:74-109.

15. Leiter L, Yale J, Chiasson J, et al. Assessment of the impact of fear of hypoglycemic episodes on glycemic and hypoglycemia management. Diabetes 2005;29:186-92.

16. American Diabetes Association. Standards of Medical Care in Diabetes-2017. Diabetes Care 2017;40(S10.

17. van Beers CAJ, DeVries JH. Continuous Glucose Monitoring. J Diabetes Sci Technol 2016;10:1251-8.

18. Cryer P. Hypoglycemia in diabetes: pathophysiology, prevalence, and prevention. 2nd edn. United States of America: American Diabetes Association, Inc, 2012.

19. Elliott L, Fidler C, Ditchfield A, et al. Hypoglycemia Event Rates: A Comparison Between Real-World Data and Randomized Controlled Trial Populations in Insulin-Treated Diabetes. Diabetes Ther 2016;7:45-60.

20. Leiter LA, Boras D, Woo VC. Dosing irregularities and self-treated hypoglycemia in type 2 diabetes: results from the Canadian cohort of an international survey of patients and healthcare professionals. Can J Diabetes 2014;38:38-44.

21. Geelhoed-Duijvestijn PH, Pedersen-Bjergaard U, Weitgasser R, et al. Effects of patient-reported non-severe hypoglycemia on healthcare resource use, work-time loss, and wellbeing in insulin-treated patients with diabetes in seven European countries. J Med Econ 2013;16:1453-61.

22. Östenson CG, Geelhoed-Duijvestijn P, Lahtela J, et al. Self-reported non-severe hypoglycaemic events in Europe. Diabetic Medicine 2014;31:92-101.

23. Lüddeke HJ, Sreenan S, Aczel S, et al. PREDICTIVE- a global, prospective observational study to evaluate insulin detemir treatment in types 1 and 2 diabetes: baseline characteristics and predictors of hypoglycaemia from the European cohort. Diabetes Obes Metab 2007;9:428-34.

24. Munro N, Barnett AH. Incidence, worry and discussion about dosing irregularities and self-treated hypoglycaemia amongst HCPs and patients with type 2 diabetes: results from the UK cohort of the Global Attitudes of Patient and Physicians (GAPP2) survey. Int J Clin Pract 2014;68:692-9.

25. Brod M, Wolden M, Christensen T, et al. Understanding the economic burden of nonsevere nocturnal hypoglycemic events: impact on work productivity, disease management, and resource utilization. Value Health 2013:16:1140-9.

26. Brož J, Brabec M, Janíčková Žd'árská D, et al. Fear of driving license withdrawal in patients with insulin-treated diabetes mellitus negatively influences their decision to report severe hypoglycemic events to physicians. Patient Prefer Adherence 2015;9.

27. Smith $C B$, Choudhary P, Pernet $A$, et al. Hypoglycemia unawareness is associated with reduced adherence to therapeutic decisions in patients with type 1 diabetes: evidence from a clinical audit. Diabetes Care 2009;32:1196-8.

28. Sarkar U, Karter AJ, Liu JY, et al. Hypoglycemia is more common among type 2 diabetes patients with limited health literacy: the Diabetes Study of Northern California (DISTANCE). J Gen Intern Med 2010;25:962-8.

29. Mansi I. Statin Adverse Events in Primary Prevention: Between Randomized Trials and Observational Studies. Am J Med Sci 2015;350:330-7.
30. Ratzki-Leewing A, Harris S, Ryan B, et al. Hypoglycemia Rates in Type 2 Diabetes Mellitus: results of the Population-based InHypoDM Study. Can J Diabetes. In Press. 2017.

31. Reichert S, Harris S, Ryan B, et al. A Population-Based Study on Incidence and Associated Risk Factors for Hypoglycaemia in Canada: The InHYPO-DM Study. Can J Diabetes 2016;59(Suppl1):S394-5.

32. Harris S, Reichert S, Ryan BL, et al. A Population-Based Study on Incidence and Associated Risk Factors for Hypoglycemia in Canada: The InHYPO-DM Study. Can J Diabetes 2016;40:S11-S12.

33. Reichert S, Harris S, Mequanint S, et al. A National Survey of Physicians' and Allied Health Professionals' Practices and Perspectives Regarding Hypoglycemia Management: The InHYPODM Studylmage 8. Can J Diabetes 2016;40:S58-S59.

34. Ratzki-Leewing A, Harris S, Ryan B, et al. A Survey of Canadians with Diabetes: Insight into Hypoglycemia Management (The InHYPO-DM Study). Can J Diabetes 2016;65:A105.

35. Ipsos Interactive Services Limited. Ipsos answers to ESOMAR 28 questions to help online research buyers. Paris, France: Ipsos, 2015.

36. Cheng AY. Canadian Diabetes Association Clinical Practice Guidelines Expert Committee. Canadian Diabetes Association 2013 clinical practice guidelines for the prevention and management of diabetes in Canada. Introduction. Can J Diabetes 2013;37 Suppl 1:S1-212

37. U.S. Department of Health and Human Services FDA Center for Drug Evaluation and ResearchU.S. Department of Health and Human Services FDA Center for Biologics Evaluation and ResearchU.S. Department of Health and Human Services FDA Center for Devices and Radiological Health. Guidance for industry: patient-reported outcome measures: use in medical product development to support labeling claims: draft guidance. Health Qual Life Outcomes 2006;4:79.

38. Costello A, Osborne J. Best practices in exploratory factor analysis: four recommendations for getting the most from your analysis. Practical Assessment, Research \& Evaluation 2005;10:173-8.

39. Statistics Canada. Diabetes, by sex, provinces, and territories. Canada: Statistics Canada, 2016.

40. Foos V, Varol N, Curtis BH, et al. Economic impact of severe and non-severe hypoglycemia in patients with Type 1 and Type 2 diabetes in the United States. J Med Econ 2015;18:420-32.

41. Aronson R, Goldenberg R, Boras D, et al. The Canadian Hypoglycemia Assessment Tool Program: Insights Into Rates and Implications of Hypoglycemia From an Observational Study. Can J Diabetes 2018;42:11-17.

42. Salutini E, Bianchi C, Santini M, et al. Access to emergency room for hypoglycaemia in people with diabetes. Diabetes Metab Res Rev 2015;31:745-51.

43. Monami M, Dicembrini I, Kundisova L, et al. A meta-analysis of the hypoglycaemic risk in randomized controlled trials with sulphonylureas in patients with type 2 diabetes. Diabetes Obes Metab 2014;16:833-40.

44. Schopman JE, Simon AC, Hoefnagel SJ, et al. The incidence of mild and severe hypoglycaemia in patients with type 2 diabetes mellitus treated with sulfonylureas: a systematic review and meta-analysis. Diabetes Metab Res Rev 2014;30:11-22.

45. Government of Canada SC. Internet use by individuals, by selected characteristics. Canada: Government of Canada SC, 2010.

46. Seaquist E, Anderson J, Childs B, et al. Hypoglycemia and diabetes: a report of a workgroup of the American Diabetes Association and the Endocrine Society. 2013;36:1384-95.

47. Khunti K, Alsifri S, Aronson R, et al. Rates and predictors of hypoglycaemia in 27585 people from 24 countries with insulintreated type 1 and type 2 diabetes: the global HAT study. Diabetes Obes Metab 2016;18:907-15

48. Jaap AJ, Jones GC, McCrimmon RJ, et al. Perceived symptoms of hypoglycaemia in elderly type 2 diabetic patients treated with insulin. Diabetic Medicine 1998;15:398-401.

49. Gabriely I, Shamoon H. Hypoglycemia in diabetes: common, often unrecognized. Cleve Clin J Med 2004;71:335-42.

50. Pramming S, Thorsteinsson B, Bendtson I, et al. Symptomatic hypoglycaemia in 411 type 1 diabetic patients. Diabet Med 1991;8:217-22.

51. Pedersen-Bjergaard U, Pramming S, Thorsteinsson B. Recall of severe hypoglycaemia and self-estimated state of awareness in type 1 diabetes. Diabetes Metab Res Rev 2003;19:232-40. 